\title{
Engaging Community Pharmacies in Early Detection of Missing Tuberculosis Patients through Public-Private Mix Intervention in Pakistan
}

\author{
Waseem Ullah, ${ }^{1,2,3}$ Hadi Almansour, ${ }^{3}$ Razia Fatima, ${ }^{4}$ Bandana Saini, ${ }^{3,5}$ and Gul Majid Khan ${ }^{1 *}$ \\ ${ }^{1}$ Department of Pharmacy, Quaid-i-Azam University, Islamabad, Pakistan; ${ }^{2}$ Greenstar Social Marketing Pakistan Guarantee Limited, Lahore, \\ Pakistan; ${ }^{3}$ School of Pharmacy, Faculty of Medicine and Health, University of Sydney, Sydney, Australia; ${ }^{4}$ Chief Research Unit, National \\ Tuberculosis Control Program, Islamabad, Pakistan; ${ }^{5}$ The Woolcock Institute of Medical Research, Sydney, Australia
}

\begin{abstract}
Globally, Pakistan ranks fifth in terms of missing tuberculosis (TB) patients' burden. Missed TB cases are either undiagnosed or diagnosed but not notified to the national TB database. Public-private mix interventions are contributing significantly to the case detection, diagnosis, and treatment of TB in Pakistan. However, it is estimated that many cases of infected TB patients go undetected. It is likely that these "undiagnosed" active TB cases seek treatment from community pharmacies, among other venues. This study aimed at assessing the feasibility of community pharmacy-based TB case detection. Case detection protocol implementation in three Pakistani districts in a nonrandom selection of pharmacies was followed by a review of routinely maintained prospective records of patients referred from these private community pharmacies to general practitioner (GP) clinics. The study engaged 500 community pharmacies for referring presumptive TB patients to GP clinics. In total, $85 \%$ of the engaged pharmacies remained active in providing referrals during the study period. The community pharmacy-referral network achieved an annual referral rate of 3,025 presumptive TB patients and identified 547 active TB cases for the period January-December 2017. Every fifth referral among presumptives presenting and counseled at pharmacies was diagnosed with TB at GP clinics. This contribution was $9 \%$ of all new TB cases identified in these districts through all other private venues linked with the Greenstar Social Marketing setup. Identified barriers and facilitators to implementation and cost effectiveness of pharmacy models for TB case detection should be considered if the model were to be scaled up.
\end{abstract}

\section{INTRODUCTION}

Among communicable diseases, tuberculosis (TB) imposes a serious burden on developing economies such as Pakistan. ${ }^{1}$ The WHO works with high-burden countries to create national TB control programs (NTPs) aimed at developing strategic methods to detect, treat, and control the spread of TB. ${ }^{2}$ For each country, based on various sources of data, the WHO calculates an estimated number of TB cases that likely exist in each country. When these data on "estimated" annual cases are compared with "actual" annual data, gaps are often evident and are defined as "missing TB cases" (i.e., the potential number of undiagnosed/misdiagnosed/diagnosed cases that have not been notified to national registries). ${ }^{2}$ These missed TB cases if undiagnosed contribute in transmitting TB disease in the community. ${ }^{3}$ Furthermore, patients with undiagnosed TB may be inappropriately treated. As their treatment would occur outside of the NTP-mandated guidelines, they may develop drug-resistant TB. ${ }^{4}$ Delayed diagnosis in such cases contributes to poor treatment outcomes for patients when eventually diagnosed. ${ }^{5}$ At the NTP level, underdiagnosis/ misdiagnosis leading to missed TB cases may be best understood by exploring the steps in case detection, diagnostic testing, care provision pathways at a local level. ${ }^{6}$ In India, missing TB cases exist because of a long, complex TB care pathway as these presumptive patients seek TB care from an average of three different providers over a 2-month time frame before getting diagnosed as a TB patient. ${ }^{7}$ As $84 \%$ of Pakistani population seeks health care from private sector facilities, ${ }^{8}$ private providers are less motivated to report TB cases to the NTP, ${ }^{9}$ which leads to significant under-detection of missing TB cases in Pakistan. ${ }^{1}$

*Address correspondence to Gul Majid Khan, Department of Pharmacy, Quaid-i-Azam University Islamabad, Islamabad 45320, Pakistan. E-mails: gmkhan@qau.edu.pk or drgulmajeed@yahoo.com
Globally, there are three million TB cases that are "missed" from an estimate of 10 million new TB cases expected each year. Nearly $80 \%$ of these missing TB cases are postulated to exist in 10 countries, and Pakistan ranks fifth in the globally estimated burden of missed TB cases. The TB case detection rate of Pakistan stands at $64 \%(360,472$ cases notified among 562,000 expected incident cases); in other words, the gap of $36 \%$ (201,528 missing TB patients) exists between case notification and expected case incidence. ${ }^{10}$

One of the main strategies that the WHO has instigated to eliminate between actual and expected TB case notification in 13 high-burden countries cases is the engagement of the private sector in case notification and improvement of quality in TB care. ${ }^{6}$ In developing economies, specifically Pakistan, it is estimated that $24 \%$ of all TB patients are identified and managed by private providers. ${ }^{11}$ Pakistan has cohesively engaged its private stakeholders in TB care by using the public-private mix (PPM) intervention (2014), where different types of private providers collaborate with public TB control centers. There are four common combinations of public TB care facilities with private collaborators in TB care referred to as "models" in Pakistan: solo general practitioner model (PPM-1), non-governmental organization run TB care facilities model (PPM-2), private hospital model (PPM-3), and para-statal model (PPM-4). Conventionally, PPM-1 operates through a privately owned clinic by a general practitioner (GP) supported by paramedic staff which is linked with a private laboratory through a district TB control program. ${ }^{12}$ Among PPM models, the solo GP model contributes substantially to national TB case notification. ${ }^{13}$ It is clear that despite the heavy investment in PPM, many patients are missed in Pakistan. This is possibly due to the fact that the private sector in Pakistan is huge, and in addition to the aforementioned PPM providers, there are diverse private sector TB stakeholders, including community pharmacies, who are not actively involved with the public sector NTP facilities in advancing TB case detection rates in Pakistan. ${ }^{12}$ 
There is a large network of community pharmacies in Pakistan, and consumers (presumptive TB patients, i.e., patients who likely have TB based on symptom patterns but are not yet diagnosed) may initiate TB care at these venues because of easy availability of anti-TB medication (antibiotics) at community pharmacies. ${ }^{14}$ In countries where enforcement of prescription medicine supply has low oversight because of economic restraints, consumers seldom purchase TB medicines through prescription from a healthcare provider. ${ }^{15}$ It is reported that a majority of the world population purchase antibiotics without a valid prescription from community pharmacies. ${ }^{15}$ But non-prescribed use of antibiotics is associated with irrational use of drugs in the form of incomplete, shorter treatment courses, inappropriate drug, and dosage. ${ }^{16,17}$ This approach consequently leads to a masking of or delay in the timely and accurate diagnosis of the infectious diseases including TB. ${ }^{18}$ Fluoroquinolones are a classic example, where their usage temporarily suppresses tuberculosis symptoms; thus, this results in fluoroquinolone sales to presumptive TB cases resulting in them receiving several courses of these drugs rather than antimicrobials mandated in NTP-based regimens, leading to development of multidrug-resistant tuberculosis. ${ }^{19}$ Although this practice of obtaining non-prescription TB medication at private pharmacies is fraught with danger, a large number of customers still initiate TB care from community pharmacies because of their easy access, long operating hours, and no consultation fee. ${ }^{20,21}$

Given the substantial contribution of PPM- $1,{ }^{13}$ and the large estimates of community pharmacies providing TB medications without prescription, ${ }^{14}$ a possible way to increase TB case detection rates and guideline-adherent TB care offered at private facilities may be to bring community pharmacies into the folds of the PPM-1 model. ${ }^{22}$ Based on this rationale, we designed a study to enhance the early case detection of missing TB cases within the Pakistani healthcare system by involving private pharmacies with private general practitioners and laboratories (conventional PPM-1) in a novel approach (PPM-1 boosted). The mechanism of operation of this novel model was the development of a formalized referral pathway for patients seeking TB care at pharmacies. Thus, in doing this, apart from enhancing TB case detection and lowering the mass of "missing TB cases," additional benefits include a paradigm shift in pharmacy operations-moving away from a "sales" focus to a "counseling and referral" or triage role. This clearly can reduce the undesirable effects of self-sourced antibiotics/drug-resistant TB and poorer prognosis by progressing presumptive TB cases to receive care from trained GPs following standard TB treatment guidelines.

Scoping the aforementioned aims, we also took into consideration the literature-for example, learning from experience of pilot-scale engagement of community pharmacies in a TB referral network in Pakistan ${ }^{23}$ to streamline our objectives. The specific objectives were

1. to determine differences between the sociodemographics of TB cases notified through conventional PPM-1 and PPM-1 boosted models,

2. to assess the quarterly rate of presumptive TB case referral (no of presumptive TB patients referred from community pharmacies to GP clinics each quarter),

3. to explore the possible effect of training on referral provision at community pharmacies, and

4. to elaborate the referral patterns at community pharmacies.

\section{MATERIALS AND METHODS}

Setting. The PPM-1 boosted model for case detection was implemented in three districts, namely, Gujrat, Lahore, and Sheikhupura.

Study design. The study used a method of case detection by pharmacies followed by referral, all supported by documentation. Data collection involved following up on referred patients to capture referral uptake and final outcomes.

Study population. This included all presumptive TB cases presenting at community pharmacies participating in the PPM-1 boosted model between January 2017 and December 2017.

Ethical approval. Ethical approval for the conduct of this project was obtained from the Institutional Review Board (IRB) Ethics Committee, common unit, National TB Control Program, Ministry of National Services, Regulations, and Coordination, Islamabad, Pakistan. (F. No. IRB - CMU-2019-06).

Resource allocation. The Greenstar Social Marketing (GSM) organization as a sub-recipient to the NTP obtained grant in this project, operationalized the implementation of PPM models for provision of directly observed treatment short course (DOTS) regionally. To achieve this, GSM had registered with them a network of private general practitioner clinics and laboratories. Greenstar Social Marketing also hired and trained facilitators at three levels (cluster, district, and region) for the purposes of auditing and operationalizing PPM-1 models. Our project used the infrastructure setup by GSM to fit the PPM-1 boosted model to recruit private pharmacies and link them with the GSM setup of PPM-1 (solo GP + public facility models).

Recruitment criteria. To be considered as eligible for an invitation to participate, pharmacies had to be

1. located within $\leq 5 \mathrm{~km}$ radius from a private GP clinic registered with GSM,

2. located within $5-10 \mathrm{~km}$ radius from a private laboratory registered with GSM,

3. have a minimum patient flow of 100 patients per day, and

4. willing to refer presumptive TB patients instead of providing medications.

The distance inclusion was based arbitrarily for convenience of patients (taking referrals to GP practices, or for quicker results) transfer between GP practices and public/ private laboratories.

Sampling frame development process. Based on the aforementioned criteria, a sampling frame to recruit potential pharmacies to participate in the PPM-1 boosted model was developed. The three districts Gujrat, Lahore, and Sheikhupura were divided into seven clusters with each cluster assigned a facilitator. Cluster facilitators directly observed the pharmacy location (as a list of pharmacies are not available through professional organizations) and patient flow as per the aforementioned criteria (1-3) and prepared a list of pharmacies in all clusters that could potentially be recruited. Once the sampling frame was developed, cluster facilitators visited each pharmacy identified in their cluster and provided verbal project information to pharmacy owners (recruitment visit). This approach was used as in the Pakistani context, given pharmacy owners, who may or may not be pharmacists, have the authority to make executive decisions about participation in health programs and to delegate staff/resources to a particular program. Pharmacists can, of course, consent to 
participate, but they can do this for their own development/ professional fulfillment but may not have the staff/resource support needed to action a particular program. If pharmacy staff-in-charge/owner was willing to adhere to project protocols (criteria 4 given earlier), then they were consented (verbally) as study participants. PPM-1-based GP clinics/ laboratory staff were also informed about likely presentation of presumptive cases referred by participating community pharmacies.

Training. Brief initial training (Training 1): All consenting pharmacy owners (or staff-in-charge) were provided with on the spot training at the recruitment visit by cluster facilitators about TB symptoms, national TB treatment guidelines, and maintaining the referral register for presumptive patients and were given a hard copy directory of GP clinics and private laboratories located within a 5- to $10-\mathrm{km}$ radius of the pharmacy. Pharmacy owners were asked to nominate any one of the pharmacy staff to be additionally trained.
Comprehensive training (Training 2): A second detailed training was then conducted at venues outside the recruited community pharmacies (travel allowance provided to participants). Signed consent (written) was obtained from all attendees to participate in the PPM-1 boosted model implementation. The training program involved a didactic presentation on disease epidemiology, high-risk population, and pharmacy staff role in the early case detection among presumptive TB patients, followed by a detailed question-answer session. Printed brochures and tool kits designed with project key points were handed to pharmacy staff for display and as reference materials.

Implementation and auditing. In each pharmacy, potential project participants included the pharmacy owners, pharmacists, and pharmacy assistants; all were invited to participate in training (either training session 1 or 2 or both) as other project meetings outlined ahead. Once trained, participating pharmacy staff then progressed to the case identification

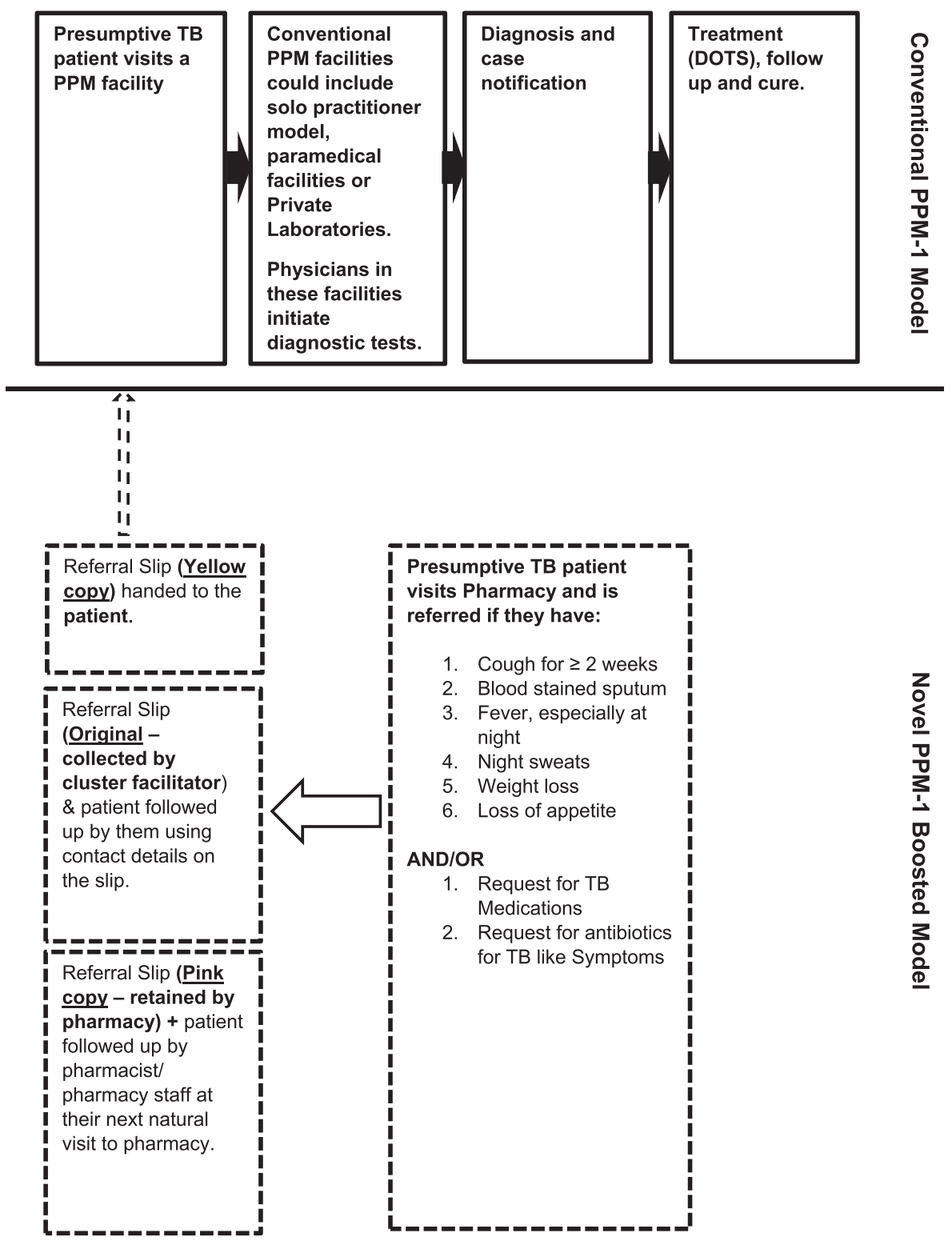

FIGURE 1. Conventional PPM-1 model and PPM-1 boosted model. PPM = public-private mix. 
phase. In this phase, patients were deemed to be presumptive TB cases if they demonstrated any of the six main symptoms of TB disease or were purchasing anti-TB medication without prescription (as shown in Figure 1). These case patients were informed about the project, and consent for their participation was sought verbally. If the patient consented to participate in the study verbally, a referral slip was filled out by pharmacy staff. This referral slip identified the patient's name, contact details, and symptoms along with the contact details of the PPM-1-based GP clinic being referred to and the names of the referring community pharmacy and the cluster representative. Each referral slip was designed with two auto-generated carbon copies, a pink and a yellow copy. The yellow copy was provided to the patient for handing over to the GP clinic; the original referral slip was collected by the cluster facilitator during weekly visits to each participating pharmacy, and details were used to follow up patients not reporting at PPM-1-based GP clinics. The conversations of cluster facilitators with referrals who did not visit GP clinics were not formally recorded or documented, but cluster facilitators were encouraged to diarize key findings. At subsequent pharmacy presentations by patients who had not taken up the referral, participating pharmacy staff provided counseling using the retained pink copies. Fortnightly visits to each pharmacy participating in this PPM-1 boosted model were also conducted by regional facilitators. The key issues from the conversations of cluster facilitators with pharmacies staff and patients as noted by them were discussed with GSM and NTP stakeholders during monthly and quarterly project review meetings.

Monitoring and evaluation of the project/community pharmacy-referral network involved monthly meeting with GSM stakeholders (regional coordinator) and quarterly review meetings with NTP stakeholders (district TB coordinator). In addition, random monitoring visits were conducted by GSM and NTP stakeholders in any of the clusters and districts within the region throughout the project. Local participating community pharmacy staff were invited to attend these meetings.

Data analysis. The collected data were entered into Excel (Microsoft Excel 2016 edition). After cleaning, data were checked for accuracy of entry and then analyzed descriptively for the study period for the process indicators and outcomes reported in Table 1. The data were also compared against the performance of the conventional PPM-1 model for the same time period-obtained through the national TB register.

Implementation approach. In planning the implementation of the PPM-1 boosted project, the COM-B model was adopted, and likely behavioral barriers during implementation were addressed to as much an extent as possible ${ }^{24}$ (see Table 2).

\section{RESULTS}

A total of 500 pharmacies consented to participate in testing the PPM-1 boosted model (PPM-B) between January and December 2017 in the three districts (Gujrat, Lahore, and Sheikhupura). These pharmacies resulted in achieving a pharmacy annual referral rate of 3,025 presumptive TB patients. From these referrals, 1,901 patients in receipt of a referral actually visited GP clinics (referral uptake $=63 \%$ ) and 547 cases were diagnosed with TB, that is, a positive referral outcome of $18 \%$ (see Figure 2 for a detailed breakup of referrals per district). Overall, the referrals registration as TB cases (presumptive cases referred/TB cases diagnosed) was 5.5 (3.6 for Gujrat, 5.3 for Lahore, and 7.0 for Sheikhupura).

Notes taken by cluster facilitators during telephone followup of TB presumptive cases unwilling to take up the referral revealed that some of these patients (i.e., $37 \%$ of those provided a referral) had previously visited the GP clinics to which they had been referred and were not convinced about visiting the GP again; their reasons for this reluctance were not often disclosed. Some of the referred cases not acting on the referral reported that GPs requested fees on their (patients') first visit to the GP clinic (before this project); possibly this apprehension was one reason for not attending GP clinics and acting on the referral in the current project. During quarterly review meetings, where some of the participating pharmacies attended, the fact that consultation fee may be a barrier to convince some presumptive TB cases referred by them was also mentioned.

TABLE 1

Key definitions of community pharmacies' referral network

\begin{tabular}{|c|c|c|}
\hline Indicator & Definition & Source \\
\hline $\begin{array}{l}\text { Referral uptake } \\
\text { (primary outcome) }\end{array}$ & $\begin{array}{l}\text { The actual number of presumptive TB cases visiting } \\
\text { the GP clinic after pharmacy referral/total cases } \\
\text { referred from community pharmacies }\end{array}$ & $\begin{array}{l}\text { Obtained by matching yellow referral slips collected } \\
\text { from PPM-1 clinics to all original slips collected } \\
\text { from participating pharmacies }\end{array}$ \\
\hline Referral delay & $\begin{array}{l}\text { The time period between detailed training (Training } \\
\text { 2) of pharmacy staff and the first referral from } \\
\text { community pharmacy }\end{array}$ & By noting the earliest date on an original referral slip \\
\hline Diagnostic delay & $\begin{array}{l}\text { The time period between referral provision to the } \\
\text { patient and the patient's registration at the GP } \\
\text { clinic for notified TB cases }\end{array}$ & $\begin{array}{l}\text { By noting the date on an original referral slip and date } \\
\text { at which a particular patient reported at a } \\
\text { participating PPM- } 1 \text { clinic }\end{array}$ \\
\hline $\begin{array}{l}\text { Referral outcome } \\
\text { (secondary outcome) }\end{array}$ & $\begin{array}{l}\text { The total number of TB cases notified/total cases } \\
\text { referred from community pharmacies } \times 100\end{array}$ & $\begin{array}{l}\text { By looking at the national case notification register } \\
\text { and matching patients with those referred from the } \\
\text { three districts }\end{array}$ \\
\hline $\begin{array}{l}\text { Referrals generated } \\
\text { from brief training }\end{array}$ & $\begin{array}{l}\text { The number of referrals provided by pharmacies } \\
\text { after "on the spot" (Training } 1 \text { [brief]) but before the } \\
\text { second detailed training (Training 2) }\end{array}$ & $\begin{array}{l}\text { By noting the dates on original referral slips and } \\
\text { comparing with training dates for a particular } \\
\text { pharmacy }\end{array}$ \\
\hline $\begin{array}{l}\text { Referrals generated } \\
\text { from full training }\end{array}$ & $\begin{array}{l}\text { The number of referrals provided by pharmacies } \\
\text { after the second detailed training (Training 2) }\end{array}$ & $\begin{array}{l}\text { By noting the dates on original referral slips and } \\
\text { comparing with training dates for a particular } \\
\text { pharmacy }\end{array}$ \\
\hline Pharmacy annual referral rate & $\begin{array}{l}\text { The number of referrals made per pharmacy across } \\
\text { the year (January-December 2017) in which the } \\
\text { project was run }\end{array}$ & $\begin{array}{l}\text { By counting all referrals made by each pharmacy } \\
\text { (using original slips collected by cluster } \\
\text { facilitators) }\end{array}$ \\
\hline
\end{tabular}


TABLE 2

Application of the COM-B model to facilitate PPM-1 boosted implementation

\begin{tabular}{|c|c|c|}
\hline $\begin{array}{c}\text { COM-B model key } \\
\text { behavioral determinants }\end{array}$ & $\begin{array}{l}\text { Behavioral subtype that } \\
\text { may affect implementation }\end{array}$ & Practical strategies preplanned to overcome pharmacy staff/pharmacists' behavioral barriers to implementation \\
\hline \multirow[t]{2}{*}{ Capability } & Physical & $\begin{array}{l}\text { Providing pharmacists/pharmacies with project implementation resources allowing for } \\
\text { documentation and referral, for example, directory of PPM- } 1 \text { clinics in the area }\end{array}$ \\
\hline & Psychological & Comprehensive training which included troubleshooting \\
\hline \multirow[t]{2}{*}{ Motivation } & Reflective & $\begin{array}{l}\text { Weekly visits by cluster facilitators to each participating pharmacy to engage with } \\
\text { participants and help "facilitate" any plans/ideas or mitigate any problems }\end{array}$ \\
\hline & Automatic & $\begin{array}{l}\text { Provision of certification on training, invitation to attend quarterly review meetings, } \\
\text { announcing the top five pharmacies (referral numbers) at these meetings }\end{array}$ \\
\hline \multirow[t]{2}{*}{ Opportunity } & Physical & Providing travel allowance for pharmacy staff to attend Training 2. \\
\hline & Social & $\begin{array}{l}\text { Informing GPs to expect referrals and letting participants know that GPs in the area } \\
\text { would be expecting referrals-to enhance the social acceptance of pharmacists } \\
\text { providing non-dispensing services }\end{array}$ \\
\hline
\end{tabular}

The PPM-1 boosted model (pharmacy referrals to boost conventional PPM-1 model) contribution toward the detection of missing TB cases was $9 \%$ in the operational districts of the traditional PPM-1 model during the study period. Table 3 highlights that sociodemographics of the 547 cases registered through the PPM-1 boosted model as diagnosed TB cases. Of these cases, 53\% were male; in other words, the gender ratios were nearly equal. This also matches the gender profile of notified TB cases emerging from the conventional PPM-1 model, where the male-to-female ratio is $50 \%$ each as well. However, the TB case notifications in the age-group 35-44 years were significantly higher proportionally through the PPM-1 boosted model (e.g., patients diagnosed after pharmacy referral) as opposed to the conventional PPM-1 model (33\% versus $28 \%$ ). The proportion of TB cases younger than 15 years of age was significantly lower in the PPM-1 boosted model than the conventional PPM-1 model (6\% versus $10 \%)$.

Pulmonary TB case identification also appeared to be significantly higher in the PPM-1 boosted (80\%) than the conventional PPM-1 (73\%) compared with extrapulmonary TB case detection. Only $20 \%$ of TB patients identified were tested as bacteriologically positive in the PPM-1 Boosted model; this proportion was (27\%) in the conventional PPM-1 model. Clearly, in both models, clinically diagnosed TB cases were predominant. The PPM-1 boosted model's contribution was significantly higher ( $P$-value: 0.04$)$ in identifying relapsed TB cases and significantly lower ( $P$-value: 0.04$)$ in new TB cases among different categories of TB patients involved.

After the implementation of the PPM-1 boosted model in all three operational districts, an interesting trend of referrals was
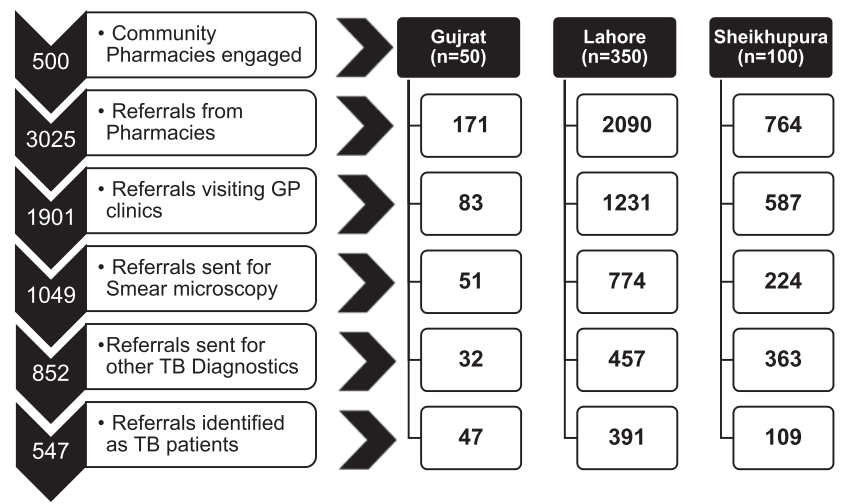

FIGURE 2. Flow diagram of referrals from community pharmacies in Gujrat, Lahore, and Sheikhupura. recorded, from 7\% in Quarter 1 to 24\% in Quarter 2 until 38\% in Quarter 3 and then dropped to 31\% in Quarter 4. The Quarter 4 referral decline was highest in Gujrat pharmacies (from $34 \%$ in Quarter 3 to 17\% in Quarter 4). (see Table 4 for referrals details in all four quarters). All the cluster facilitators were regularly performing the weekly visits to the recruited pharmacies throughout all four quarters in Lahore and Sheikhupura districts except Gujrat where the cluster facilitator continued weekly visits till Quarter 3 but resigned after the quarterly review meeting in the end of September. After this point, only fortnight visits were performed by the regional facilitator to the recruited pharmacies in Gujrat during Quarter 4.

As highlighted in the Methods section, a brief initial onsite training was provided at the start of the project. During Quarter 1,50 pharmacies in Gujrat, 350 pharmacies in Lahore, and 100 pharmacies in Sheikhupura had received this training. The comprehensive training for all the recruited pharmacies (Training 2) was initiated by the end of Quarter 1 (February 27, 2017) and concluded in the latter half of Quarter 2 (June 6, 2017). Post-comprehensive training, there appeared to be an increasing trend in total referrals from $14 \%$ to $71 \%$ in three districts: $24-42 \%$ in Gujrat, $10-76 \%$ in Lahore, and $21-69 \%$ in Sheikhupura. Figure 3 represents the community pharmacy referrals from Quarter 1 to 4 (i.e., pre-comprehensive training to post-comprehensive training) for the study period. Table 5 depicts that $85 \%$ pharmacies actively participated in providing referrals in three districts, although the remaining $15 \%$ of the engaged pharmacies did not contribute to the pharmacy referral rate from January to December 2017. Among the highest referring pharmacies, in terms of quarter-wise referral performance, $24 \%$ of the pharmacies in Gujrat provided referrals in only one quarter, $32 \%$ pharmacies in Lahore were active in only two quarters, and $35 \%$ pharmacies in Sheikhupura were referring patients in only three of the four quarters of the study. Across all quarters, referral activity levels were mostly in the range of 1-4 referrals/quarter-which was the case for $41 \%$ of pharmacies. Higher referral activity, that is, in the range of 5-8 referrals/quarter, was demonstrated by $21 \%$ of the participating pharmacies. Only $22 \%$ of pharmacies demonstrated highest activity, for example, 9-12 referrals/ quarter or higher. Table 5 highlights the detailed referral patterns at the recruited pharmacies in the operational districts.

\section{DISCUSSION}

This is the first large-scale project involving boosting of TB case notification by using a wider catchment of health venues 
TABLE 3

Sociodemographics of TB cases notified through conventional PPM-1 and PPM-1 boosted models

\begin{tabular}{|c|c|c|c|c|}
\hline \multirow[b]{2}{*}{ Variable } & \multicolumn{4}{|c|}{ Operational districts (Lahore, Sheikhupura, and Gujrat) } \\
\hline & $\begin{array}{l}\text { Combined result } \\
\text { (all three districts) }\end{array}$ & PPM-1, $N(\%)$ & PPM-B, $N(\%)$ & $\begin{array}{c}\text { P-value } \\
\text { (PPM-1 vs. PPM-1 boosted) }\end{array}$ \\
\hline Cases notified & 5,931 & $5,384(91)$ & $547(9)$ & \\
\hline \multicolumn{5}{|l|}{ Gender } \\
\hline Male & 2,980 & $2,689(50)$ & $291(53)$ & 0.14 \\
\hline Female & 2,954 & $2,695(50)$ & $256(47)$ & 0.14 \\
\hline \multicolumn{5}{|l|}{ Age-group (years) } \\
\hline$<15$ & 588 & $558(10)$ & $30(6)$ & $0.001^{*}$ \\
\hline $15-34$ & 2,384 & $2,174(40)$ & $210(38)$ & 0.33 \\
\hline $35-54$ & 1,700 & $1,519(28)$ & $181(33)$ & $0.01^{*}$ \\
\hline$>54$ & 1,259 & $1,133(21)$ & $126(23)$ & 0.20 \\
\hline \multicolumn{5}{|l|}{ Disease subclassification } \\
\hline Pulmonary & 4,362 & $3,922(73)$ & $440(80)$ & $0.001^{*}$ \\
\hline Extrapulmonary & 1,569 & $1,462(27)$ & $107(20)$ & $0.001^{*}$ \\
\hline \multicolumn{5}{|l|}{ Subtotal pulmonary } \\
\hline Bacteriologically confirmed (B+) & 863 & $784(20)$ & $79(18)$ & 0.30 \\
\hline Non-bacteriologically confirmed (B-) & 3,499 & $3,138(80)$ & $361(82)$ & 0.30 \\
\hline \multicolumn{5}{|l|}{ TB history } \\
\hline New (Cat-1) & 5,543 & $5,043(94)$ & $500(91)$ & $0.04^{*}$ \\
\hline Relapse (Cat-II) & 388 & $341(6)$ & $47(9)$ & $0.04^{*}$ \\
\hline
\end{tabular}

TB = tuberculosis; PPM = public-private mix

* Statistically significant $P$-value.

and the allied health staff in conjunction with a conventional PPM model in Pakistan. The referral network of 500 community pharmacies in the three districts over 12 months resulted in contributing to nearly a tenth of all TB cases registered (through GSM) from these three districts in the same time period. Given the consequences of missing TB cases, this is quite a significant outcome. Statistically, our study had $80 \%$ power (at a 5\% significance level) with respect to the primary outcome (referral uptake) of the project. The results suggest that nationally, increased investment in setting up venues such as community pharmacies as part of a case detection network has value. The project protocols used implementation science principles to keep participating community pharmacy venues/staff motivated as well as an intricate system of recording outcomes. These methods possibly underpinned successful implementation and positive results that eventuated. Clearly, future PPM models to identify presumptive TB cases and progress them toward diagnosis and treatment initiation should use careful groundwork and documentation methods to elicit similar outcomes.

In this project, $85 \%$ of the 500 pharmacies recruited were actively participating in providing presumptive TB case referrals to the nearest GP clinics. This participation level is substantially higher than for pharmacies participating in TB case detection in other countries such as in Vietnam, ${ }^{25}$ Bolivia $^{26}$ Cambodia, ${ }^{27}$ Dominican Republic, ${ }^{28}$ and a previous pilot-scale project in Pakistan. ${ }^{23}$ In our project, every fifth referral from actively participating (85\%) pharmacies was converted into a diagnosed TB case at GP clinics. This finding is generally consistent with community pharmacies' engagement in TB case detection in India. In an Indian pharmacy-based TB case detection study, 81\% pharmacies of the total recruited participated, 1,674 referrals were made, and 255 cases diagnosed (i.e., every sixth referral). ${ }^{29}$ The slightly enhanced performance in our study (4\% higher rate of pharmacy active participation and every fifth referral registration as TB case) might be due to the well-worked recruitment criteria we used, the detailed method of setting up the sampling frame for potential pharmacies' involvement, and addressing of key behavioral determinants for the pharmacy/pharmacist behavior change (COM-B model) ${ }^{24}$ adopted in our study. Both the findings in the our study and the neighboring Indian pharmacy-based TB case detection project $^{29}$ indicate that increased active participation of pharmacies can likely improve the level of diagnosed TB cases and thereby decrease undiagnosed TB in the community.

However, the referral uptake of $63 \%$ in our study is rather lower than the substantial $96 \%$ referral uptake by Indian patients who were provided a referral to a PPM facility in the Indian pharmacy-based TB case detection project. ${ }^{29}$ This might be due to extended involvement of the Indian pharmacies in presumptive TB case referral and screening services in (Patna district), whereas pharmacies in our study in the three Pakistani districts were limited to referral activities only. Another systemic reason for the lower uptake of referrals by patients in our study as mentioned earlier in the results could be the apprehension about having to pay consultation fees to the GP in the PPM-1 registered clinics. This finding of consultation fee barrier was consistent with implementation of the PPM model for TB treatment in six towns in Karachi. ${ }^{9}$ This may

TABLE 4

Proportion of presumptive tuberculosis patients referred from community pharmacies to general practitioner clinics

\begin{tabular}{lcccc}
\hline \multicolumn{1}{c}{ Variable } & Total referrals & Gujrat, $N(\%)$ & Lahore, $N(\%)$ & Sheikhupura, $N(\%)$ \\
\hline Total quarters (January 2017-December 2017) & $3,025(100)$ & $171(100)$ & $2,090(100)$ & $764(100)$ \\
Quarter 1 (January 2017-March 2017) & $199(7)$ & $29(17)$ & $117(6)$ & $53(7)$ \\
Quarter 2 (April 2017-June 2017) & $737(24)$ & $55(32)$ & $507(24)$ & $175(23)$ \\
Quarter 3 (July 2017-September 2017) & $1,138(38)$ & $58(34)$ & $823(39)$ & $257(34)$ \\
Quarter 4 (October 2017-December 2017) & $951(31)$ & $29(17)$ & $643(31)$ & $279(36)$ \\
\hline
\end{tabular}




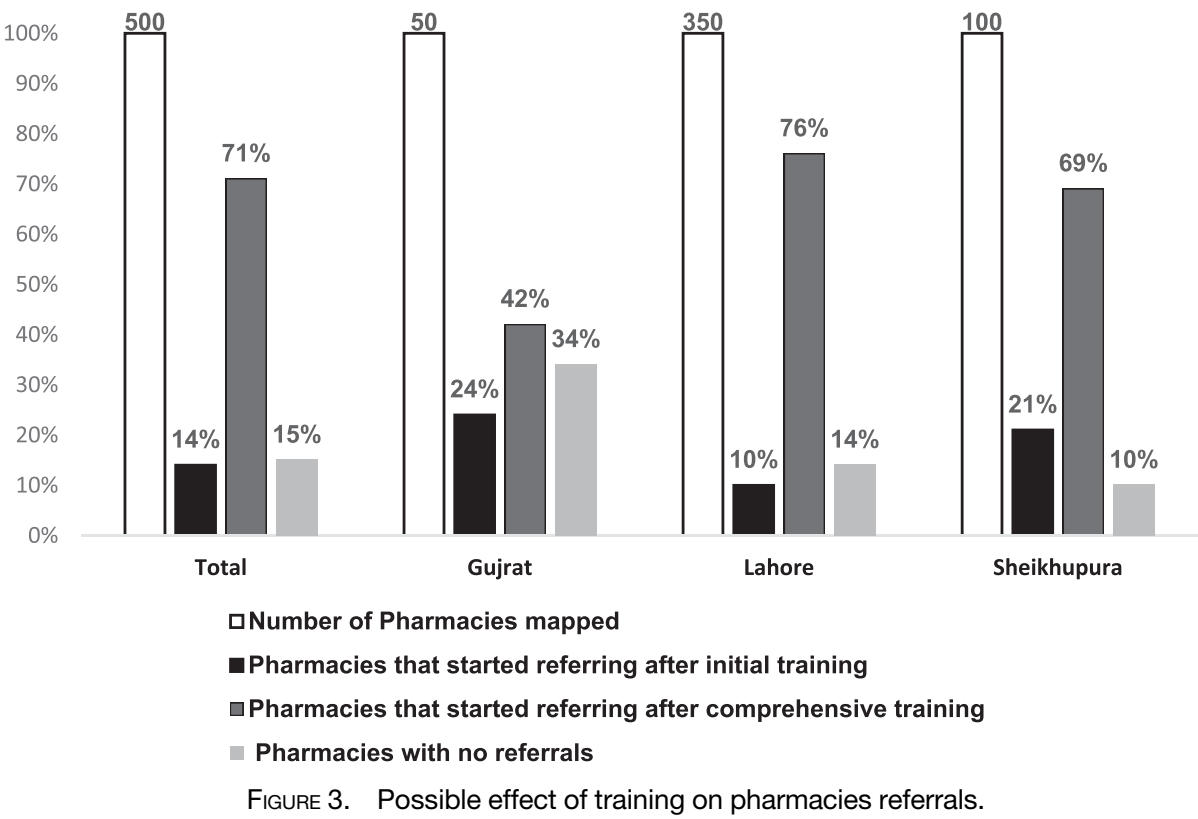

be because in the PPM model, TB treatment is provided free in a clinic only if a patient is diagnosed with TB. Otherwise, privately practicing GPs working as a part of the PPM model are free to charge consultation fees for their time expended in reviewing the case and clinical examination. Among case referrals presenting at GP clinics in our study, 45\% (852 of 1,901 referrals, Figure 2) were either referred for TB diagnostic tests excluding smear microscopy or considered non-symptomatic for TB. These symptomatic referrals might be chain-smokers or those having respiratory symptoms such as allergic cough, asthma, and chronic obstructive pulmonary disease. Thus, pharmacists' skills in identifying presumptive TB cases can be improved by updating the comprehensive training content to include awareness and differentiating factors between TB and other respiratory disease symptoms to refine the referral efficacy.

Exploring the differences between sociodemographics of TB cases identified in the conventional PPM-1 model versus our novel PPM-1 boosted model may highlight better ways to target particular cases in the future. There was no significant difference between the male-to-female ratio of TB cases notified by both PPM-1 and PPM-1 boosted models. This same ratio (1:1) of male to female TB cases notified by both the models in our study is different from a previous study in Pakistan which notified TB cases more in female than male gender (2:1). ${ }^{30}$ However, middle-aged TB patients were

TABLE 5

Elaborating the referral patterns at community pharmacies

\begin{tabular}{|c|c|c|c|c|}
\hline & Total & Gujrat & Lahore & Sheikhupura \\
\hline Variable & $N(\%)$ & $N(\%)$ & $N(\%)$ & $N(\%)$ \\
\hline Total number of pharmacies mapped & $500(100)$ & $50(100)$ & $350(100)$ & $100(100)$ \\
\hline \multicolumn{5}{|l|}{ Referral rate } \\
\hline $\begin{array}{l}\text { No. of pharmacies with }<1 \text { referral in all } \\
\text { four quarters }\end{array}$ & $77(15)$ & $17(34)$ & $50(14)$ & $10(10)$ \\
\hline $\begin{array}{l}\text { No. of pharmacies with } \geq 1 \text { referral in all } \\
\text { four quarters }\end{array}$ & $31(6)$ & $3(6)$ & $20(6)$ & $8(8)$ \\
\hline $\begin{array}{l}\text { No. of pharmacies with } \geq 1 \text { referral in } \\
\text { only three quarters }\end{array}$ & $117(23)$ & $7(14)$ & $75(21)$ & $35(35)$ \\
\hline $\begin{array}{l}\text { No. of pharmacies with } \geq 1 \text { referral in } \\
\text { only two quarters }\end{array}$ & $155(31)$ & $11(22)$ & $113(32)$ & $31(31)$ \\
\hline $\begin{array}{l}\text { No. of pharmacies with } \geq 1 \text { referral in } \\
\text { only single quarter }\end{array}$ & $120(24)$ & $12(24)$ & $92(26)$ & $16(16)$ \\
\hline \multicolumn{5}{|l|}{ Referral frequency } \\
\hline $\begin{array}{l}\text { Pharmacies referring no presumptives } \\
\text { in all four quarters }\end{array}$ & $77(15)$ & $17(34)$ & $50(14)$ & $10(10)$ \\
\hline $\begin{array}{l}\text { Pharmacies referring } 1-4 \text { presumptives } \\
\text { in all four quarters }\end{array}$ & $204(41)$ & $20(40)$ & $146(42)$ & $38(38)$ \\
\hline $\begin{array}{l}\text { Pharmacies referring } 5-8 \text { presumptives } \\
\text { in all four quarters }\end{array}$ & $105(21)$ & $5(10)$ & $80(23)$ & $20(20)$ \\
\hline $\begin{array}{l}\text { Pharmacies referring 9-12 } \\
\text { presumptives in all four quarters }\end{array}$ & $57(11)$ & $6(12)$ & $38(11)$ & $13(13)$ \\
\hline $\begin{array}{l}\text { Pharmacies referring }>12 \\
\text { presumptives in all four quarters }\end{array}$ & $57(11)$ & $2(4)$ & $36(10)$ & $19(19)$ \\
\hline
\end{tabular}


significantly contributed more by the PPM-1 boosted model (see Table 3). The working class in Pakistan mainly belongs to the (middle) age-group of 35-54 years who may prefer pharmacies because of easy access and long opening hours. Pediatric TB cases were significantly contributed less by the PPM-1 boosted model, suggesting that parents may be more conscious about health of their young-aged children and hence prefer GPs over pharmacies for TB services. These agebased patterns of the referral, therefore, reflect the decision makers' health-seeking preferences rather than the participating pharmacists' focus on those of employable age when counseling and referring.

Pulmonary cases were identified as TB presumptives for referral to a much greater extent than extrapulmonary cases in the PPM-1 boosted model (Table 3). This contribution of our boosted model was significantly higher (2.7 times) than the contribution made by the conventional PPM-1 model. These data suggest that pharmacists can more accurately pick up pulmonary TB cases. Training in future projects should, therefore, also include focused sections on symptoms in extrapulmonary TB. General practitioners referred only $55 \%$ of patients presenting at their clinics for sputum examination; this might be one of the possible reasons for contribution of more clinically diagnosed and less bacteriologically confirmed cases among pulmonary cases. This finding reflects the under-use of smear microscopy and overreliance on radiography by GPs working as a part of PPM providers in Pakistan ${ }^{31}$ and other countries. ${ }^{32}$ Preference of radiological examination over sputum examination might be due to its probable consideration as a noninvasive diagnostic approach by GPs. The PPM-1 boosted model's contribution in comparison to the PPM-1 model was significantly low in identifying new missing TB cases but significantly higher in terms of notifying previously treated patients with recurrent $T B$. Such relapsed TB patients' preference for visiting pharmacies instead of seeking TB treatment from the previous facility is concerning. This forms the basis of suggesting pharmacy staff involvement as DOTS therapy facilitators in patients with recurrent episodes of TB. Pharmacy staff can thus play a key role in improving patient adherence to TB medications, ${ }^{33}$ by offering a more convenient, accessible, and possibly a less stigmatized venue to receive TB treatment.

Quarter-wise, the lowest referral provision was recorded in Gujrat, Lahore, and Sheikhupura districts during Quarter 1 (see Table 4). This might be due to either lower participation (14\%) of pharmacies toward referrals after the brief initial training (see Figure 3 ) or the lag in setting up the process of a new implementation approach at community pharmacies. The referral uptake was increased more than three times in the operational districts during Quarter 2. This increase might be attributed to comprehensive training initiation in March and its continuation till June and the increased participation (71\%) of pharmacies in referral provision as a possible result of this training. The comprehensive training was conducted for pharmacies in their respective operational districts. A total of 28 training sessions (four in Gujrat, 16 in Lahore, and eight in Sheikhupura) were conducted over a period of 4 months. The 3-hour training program involved a didactic presentation on disease epidemiology (60 minutes), high-risk population (30 minutes), and the pharmacy staff's role in the early case detection among presumptive TB patients (30 minutes), followed by a detailed question-answer session (60 minutes). Printed brochures and tool kits designed with project key points were handed to pharmacy staff for display and as reference materials.

The trend of referral provision was highest during Quarter 3 and increased by $12 \%$ in comparison to Quarter 2 . This was possible because of intensive involvement of cluster facilitators in weekly and follow-up visits during Quarter 3 where these facilitators had been rather busy in arranging, conducting, and managing the training sessions during Quarter 2 . However, referral provision did not increase and even declined by $7 \%$ in the last quarter in comparison to Quarter 3. One reason for this tailing off effect could be that pharmacy staff were informed by NTP and GSM stakeholders in the quarterly review meeting in September that the PPM-1 boosted model might not be sustained after December 2017 because of funding priorities. Recruited pharmacies might have been expecting the referral mechanism and support systems available to them to be sustained for a longer period of time. This concern about sustainability has been reported previously, for example, by the stakeholder involved in a pharmacy TB referral program in Cambodia. $^{34}$ The lack of clarity about sustaining the current pharmacy referral program in the future after 2017 also may have affected the motivation of cluster and regional facilitators as they were used specifically for this project. This demotivation may have been a reason for the resignation of one of the cluster facilitators (in Gujrat) who resigned after Quarter 3. Thus, only fortnight visits were performed by the regional facilitator in Gujrat district, and hence, referral provision was reduced to half (17\%) in comparison to district contribution (34\%) in Quarter 3. This also highlights the importance of weekly visits by cluster facilitators and their ongoing liaison with pharmacies staff.

Qualitative studies should be performed to explore the reasons for variation in the pharmacy referral rate and referral frequency among the recruited pharmacies in the different districts. It is worth investigating the factors that affected the best and worst performance outcomes. For example, qualitative exploration of most active pharmacies in terms of referral uptake, the pharmacies most accurate in identifying the referrals with active TB cases, and inactive pharmacies with no referrals during the study period may highlight ways to overcome barriers or enhance facilitative factors in future projects, especially if a scaleup of community pharmacy integration to ongoing PPM models is considered in the future.

The pharmacy referral rate in the operational districts indicates that $15 \%$ of the 500 pharmacies did not refer any presumptive cases irrespective of the two layers of training (see Figure 3). Pharmacies not referring any presumptives might be either unsatisfied with services provided at the GP clinics $^{35}$ or considering the referral provision as a disincentive in terms of losing the clients. This latter may be the case as GPs examining the referrals were provided incentives if the referral patient was diagnosed with TB, in which case the diagnosed patient received TB medication free. Without this process, a presenting patient at a pharmacy may have been sold TB medications (or other antibiotics). Pharmacies in these districts (and nationally) are not provided with TB medication stock for free provision to diagnosed TB cases under the National TB Program in Pakistan. Thus, this potential loss of customer (and income) may have served as a disincentive for the non-referring pharmacies. Training about the ethical principles involved (beneficence) in referring TB patients and planned interaction of non-referring pharmacies with actively referring pharmacies may have served to motivate non- 
referrer pharmacies. ${ }^{36}$ Perhaps, sharing data on building customers' loyalty through ethical actions in patients' favor may have transitioned non-referrer pharmacies toward referring. For example, in other pharmacy-based TB case detection studies, participants reported that there was a tendency of referred patients to return to pharmacies as customers because of the goodwill earned. ${ }^{27}$ Incentivizing pharmacies for each case referral may have served to motivate non-referrers as well; this approach has also served well in other projects. ${ }^{29}$ Finally, setting up selected pharmacies as DOTS providers to diagnosed patients may be a mechanism for active involvement of pharmacies to provide referrals to presumptive TB cases. ${ }^{37}$

The strength of this study is that it provides a step forward toward transition from NTP policy recommendation to implementation in real-world settings with reference to scale up the solo GP model (PPM-1) in Pakistan. However, as with every study, our study has limitations. The lack of the ability to collect data of all symptomatic patients who presented at the recruited pharmacies during the study period was a limitation (e.g., it is neither clear what the denominator for the referred cases at each pharmacy was nor was it clear of the referred patients how many of the approached patients had consented). Cost-effectiveness of the PPM boosted model could not be performed in comparison to the ongoing PPM-1 model because of funding limitations, which should certainly be a consideration in future studies. The effectiveness of the training programs delivered to pharmacies/pharmacists, although evaluable in terms of referral activity conducted by participants, was not tested in terms of knowledge and skill set changes pre- and post-training mainly because of logistic constraints.

\section{CONCLUSION}

Community pharmacies offer a useful resource for scaling up PPM models of TB care in countries such as Pakistan with a fragmented but large private sector. An in-depth investment involving well-thought-through recruitment criteria, sampling frame development, and comprehensive training followed by setting up a referral mechanism at community pharmacies appeared to lead to a feasible approach in detecting missing TB cases in the Pakistani community/healthcare system within this project. Private general practitioners, pharmacies, and laboratories responded positively to this implementation approach. However, sustainability and non-provision of incentives to community pharmacies are potential barriers for their active participation in referring presumptive TB patients to the GP clinics. In future, extending the referral role of community pharmacies toward patient-oriented services will provide an opportunity to change the health-seeking behavior of presumptive TB patients presenting at pharmacies. Regulation of pharmacy outlets according to the national TB guidelines, in particular with reference to the sale of anti-TB medication, may also facilitate better referral provision in these settings. Studies focusing on costeffectiveness analysis of a community pharmacy referral network for presumptive TB cases to PPM clinics should be conducted in future to assess the economic viability of this approach.

Received December 17, 2019. Accepted for publication April 4, 2020.

Published online May 4, 2020.

Acknowledgments: We appreciate the efforts of Khalid Farough (general manager, Program: TB Project, GSM), Amjed Abbas (regional coordinator, GSM), Naseem Ullah (project officer, GSM), Syed Azadar Gillani (project manager, GSM), Syed Ghulam Haider (monitoring and evaluation manager, GSM), and Sobia Faisal (technical manager, GSM) for their continuous support throughout the implementation of this project. The American Society of Tropical Medicine and Hygiene (ASTMH) assisted with publication expenses.

Financial support: Funding for this project was obtained as part of a larger project from the Global Fund which was provided to the National Tuberculosis Control Program (NTP) of Pakistan. The NTP as the principal recipient further partnered with the Greenstar Social Marketing (GSM) organization to implement several projects in these districts. The GSM performed the resource allocation for hiring a team to work in all the districts.

Authors' addresses: Waseem Ullah, Department of Pharmacy, Quaidi-Azam University, Islamabad, Pakistan, School of Pharmacy, Faculty of Medicine and Health, University of Sydney, Sydney, New South Wales, Australia, and Greenstar Social Marketing Pakistan Guarantee Limited, Lahore, Pakistan, E-mail: waseem@bs.qau.edu.pk. Hadi Almansour, School of Pharmacy, Faculty of Medicine and Health, University of Sydney, Sydney, Australia, E-mail: halm7156@ uni.sydney.edu.au. Razia Fatima, Chief Research Unit, National Tuberculosis Control Program, Islamabad, Pakistan, E-mail: drraziafatima@ gmail.com. Bandana Saini, School of Pharmacy, Faculty of Medicine and Health, University of Sydney, Sydney, Australia, and The Woolcock Institute of Medical Research, Sydney, Australia, E-mail: bandana.saini@ sydney.edu.au. Gul Majid Khan, Department of Pharmacy, Quaid-i-Azam University, Islamabad, Pakistan, E-mail: gmkhan@qau.edu.pk.

\section{REFERENCES}

1. Qadeer E, Fatima R, Yaqoob A, Tahseen S, Ul-Haq M, Ghafoor A, Asif M, Streatesman M, Tiemersma EW, 2016. Population based national tuberculosis prevalence survey among adults (>15 years) in Pakistan, 2010-2011. PLoS One 11: 0148293.

2. World Health Organization, 2018. Global Tuberculosis Report. Available at: https://www.aidsdatahub.org/sites/default/files/ publication/WHO_Global_TB_Report_2018.pdf. Accessed November 9, 2019.

3. Rumman KA, Sabra NA, Bakri F, Seita A, Bassili A, 2008. Prevalence of tuberculosis suspects and their healthcare-seeking behavior in urban and rural Jordan. Am J Trop Med Hyg 79: 545-551.

4. Uplekar M, Lonnroth K, 2007. MDR and XDR - the price of delaying engagement with all care providers for control of TB and TB/ HIV. Trop Med Int Health 12: 473-474.

5. World Health Organization, 2006. Diagnostic and Treatment Delay in Tuberculosis. Available at: http://applications.emro.who.int/ dsaf/dsa710.pdf. Accessed November 9, 2019.

6. Chin DP, Hanson CL, 2017. Finding the missing tuberculosis patients. J Infect Dis 216: 675-S678.

7. Cooper A, 2019. Finding the missing millions affected by tuberculosis is one thing; treating them is another. Lancet Respir Med 7: 741.

8. Government of Pakistan, 2012. National Health Accounts, 2011-2012. Available at: http://www.pbs.gov.pk/content/ national-health-accounts-pakistan-2011-12. Accessed November 14, 2019.

9. Naqvi SA, Naseer M, Kazi A, Pethani A, Naeem I, Zainab S, Fatmi $\mathrm{S}, 2012$. Implementing a public-private mix model for tuberculosis treatment in urban Pakistan: lessons and experiences. Int J Tuberc Lung Dis 16: 817-821.

10. World Health Organization, 2019. Global Tuberculosis Report. Available at: https://www.who.int/tb/data/GTBreportCountryProfiles. pdf?ua=1. Accessed November 10, 2019.

11. Hanson C, Osberg M, Brown J, Durham G, Chin DP, 2017. Finding the missing patients with tuberculosis: lessons learned from patient-pathway analyses in 5 countries. J Infect Dis 216 (Suppl 7): S686-S695.

12. National Tuberculosis Control Program Pakistan, 2014. Annual TB Report. Available at: http://ntp.gov.pk/uploads/NTP_Annual_ Report_2014.pdf. Accessed November 14, 2019.

13. National Tuberculosis Control Program Pakistan, 2016. Annual TB Report. Available at: http://ntp.gov.pk/uploads/NTP_Annual_ Report_2016.pdf. Accessed November 14, 2019. 
14. Butt ZA, Gilani AH, Nanan D, Sheikh AL, White F, 2005. Quality of pharmacies in Pakistan: a cross-sectional survey. Int J Qual Health Care 17: 307-313.

15. Auta A, Hadi MA, Oga E, Adewuyi OE, Abdu-Aguye SN, Adeloye D, Strickland-Hodge B, Morgan DJ, 2019. Global access to antibiotics without prescription in community pharmacies: a systematic review and meta-analysis. J Infect 78: 8-18.

16. Miller R, Goodman C, 2016. Performance of retail pharmacies in low- and middle-income Asian settings: a systematic review. Health Policy Plann 31: 940-953.

17. Awad A, Eltayeb I, Matowe L, Thalib L, 2005. Self-medication with antibiotics and antimalarials in the community of Khartoum state, Sudan. J Pharm Pharm Sci 8: 326-331.

18. Satyanarayana $S$, Kwan $A$, Daniels $B$, Subbaraman R, McDowell A, Bergkvist S, Das RK, Das V, Das J, 2016. Use of standardised patients to assess antibiotic dispensing for tuberculosis by pharmacies in urban India: a cross-sectional study. Lancet Infect Dis 16: 1261-1268.

19. Chen TC, Lu PL, Lin CY, Lin WR, Chen YH, 2011. Fluoroquinolones are associated with delayed treatment and resistance in tuberculosis: a systematic review and meta-analysis. Int J Infect Dis 15: 211-216.

20. Wells WA, Uplekar M, Pai M, 2015. Achieving systemic and scalable private sector engagement in tuberculosis care and prevention in asia. PLoS Med 12: 1001842.

21. Bonadonna LV, Saunders MJ, Guio H, Zegarra R, Evans CA, 2018. Socioeconomic and behavioral factors associated with tuberculosis diagnostic delay in Lima, Peru. Am J Trop Med Hyg 98: 1614-1623.

22. Naidooa K, Gengiaha S, Singhd S, Stilloc J, Padayatchi N, 2019. Quality of TB care among people living with HIV: gaps and solutions. J Clin Tuberc Other Mycobact Dis 17: 10122.

23. Malik M, Rutta E, 2016. Engaging Private Sector Pharmacies in Pakistan to Increase Early TB Case Detection. Submitted to US Agency for International Development by Systems for Improved Access to Pharmaceuticals and Services (SIAPS) Program., Arlington, VA.

24. Michie S, van Stralen MM, West R, 2011. The behaviour change wheel: a new method for characterising and designing behaviour change interventions. Implement Sci 6: 42.

25. Lonnroth K, Karlsson M, Lan NT, Buu TN, Dieu TT, 2003. Referring TB suspects from private pharmacies to the national tuberculosis programme: experiences from two districts in Ho Chi Minh city, Vietnam. Int J Tuberc Lung Dis 7: 1147-1153.

26. Lambert ML, Delgado R, Michaux G, Vols A, Speybroeck N, Vander-Stuyf $P, 2005$. Collaboration between private pharmacies and national tuberculosis programme: an intervention in Bolivia. Trop Med Int Health 10: 246-250.

27. Bell CA, llomäki J, Pichenda K, Duncan GJ, Saini B, 2015. Referral of tuberculosis symptomatic clients from private pharmacies to public sector clinics for diagnosis and treatment in Cambodia. J Eval Clin Pract 21: 285-291.

28. Mitchell EMH, Perez-Then E, Orejel-Juarez I, Baez J, Gonzales F, Morrobel AL, Marcelino B, Kamp N, 2013. Effectiveness of interventions to increase referral of clients exhibiting TB symptoms by pharmacies and corner stores in santo Domingo, Dominican Republic. Open Infect Dis J 7: 47-53.

29. Daftary A, Satyanarayana S, Jha N, Singh M, Mondal S, Vadnais $\mathrm{C}$, Pai M, 2019. Can community pharmacists improve tuberculosis case finding? A mixed methods intervention study in India. BMJ Glob Health 4: 001417.

30. Codlin AJ, Khowaja S, Chen Z, Rahbar MH, Qadeer R, Ara I, McCormick JB, Fisher-Hoch SP, Khan AJ, 2011. Short report: gender differences in tuberculosis notification in Pakistan. Am J Trop Med Hyg 85: 514-517.

31. Pethani A, Zafar M, Khan AA, Rabbani Sana U, Ahmed S, Fatmi Z, 2015. Engaging general practitioners in public-private mix tuberculosis DOTS program in an urban area in Pakistan: need for context-specific approach. Asia Pac J Public Health 27: 984-992.

32. Hopewell PC, Pai M, Maher D, Uplekar M, Raviglione MC, 2006. International standards for tuberculosis care. Lancet Infect Dis 6: 710-725.

33. Khalili H, Karimzadeh I, Mirzabeigi P, Dashti-Khavidaki S, 2013. Evaluation of clinical pharmacist's interventions in an infectious diseases ward and impact on patient's direct medication cost. Eur J Intern Med 24: 227-233.

34. Bell CA, Duncan GJ, Eang R, Saini B, 2015. Stakeholder perceptions of a pharmacy-initiated tuberculosis referral program in Cambodia, 2005-2012. Asia Pac J Public Health 27: 2570-2577.

35. Bell CA, Eang MT, Dareth M, Rothmony E, Duncan GJ, Saini B, 2012. Provider perceptions of pharmacy-initiated tuberculosis referral services in Cambodia, 2005-2010. Int J Tuberc Lung Dis 16: 1086-1091.

36. Rogers EM, 2004. A prospective and retrospective look at the diffusion model. $J$ Health Commun 9: 13-19.

37. Khan MS, Salve S, Porter JD, 2015. Engaging for-profit providers in TB control: lessons learnt from initiatives in South Asia. Health Policy Plann 30: 1289-1295. 